\title{
A case of juvenile idiopathic scoliosis treated with physical therapy
}

\section{S S Wakista ${ }^{1}$}

Sri Lanka Journal of Child Health, 2006; 35: 99-100

(Key words: Juvenile idiopathic scoliosis, physical therapy)

\section{Introduction}

Scoliosis refers to a lateral deviation of the spine in the coronal or frontal plane ${ }^{1}$. It is a progressive condition that affects children during the active growth phase. If left untreated, the ultimate structural changes can only be corrected surgically ${ }^{2}$. Traditionally, preventing deformity has relied on splinting the child in cumbersome braces ${ }^{3}$. This is a case where a child was successfully managed using physical methods.

\section{Case report}

An 8 year old girl was observed by her parents to be walking asymmetrically. One shoulder was noticeably higher than the other and this asymmetry was noticed to increase as child grew. The child was referred for physical management to the Sahana Rehabilitation Centre at Dehiwala in February 2004.

Examination showed asymmetries in the shoulders, scapulae, waistline and pelvic region. Marked non tender muscular spasm was observed in the back of the chest on the left side. Neurological examination was normal and no abnormalities were detected in the leg lengths when assessed clinically and radiologically. There were no deformities or contractures in the lower limbs. An erect frontal radiograph of the dorso- lumbar spine showed a double curve scoliosis with convexity of the lumber curve to the left side and thoracic curve to the right. The scoliosis angle was measured at 20 degrees. No congenital or acquired anomalies of the vertebrae or ribs were present (Figure 1). On the basis of these investigations a diagnosis of juvenile idiopathic scoliosis was made.

\footnotetext{
${ }^{1}$ Senior Lecturer, Disability Studies Unit, Faculty of Medicine, University of Kelaniya
}

(Received on 29 December 2005. Accepted on 10 January 2006)

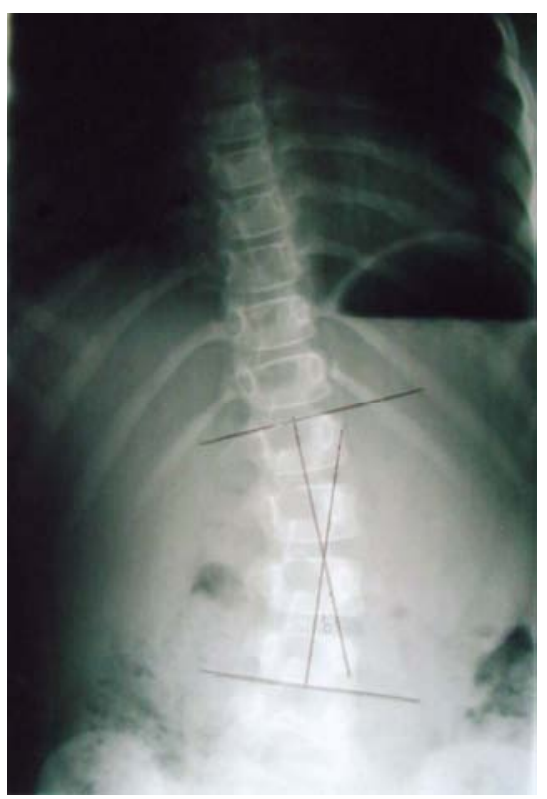

Figure 1

Management included correct positioning, relaxation of muscles by cooling and heating, and physical exercises. The child and her parents were instructed on how to maintain correct body positioning throughout the day. The exercise programme was designed to strengthen and relax the back muscles. Prior to exercises luminous infra red (IR) heat was applied to the back for 12-15 minutes, while ice massage was used to reduce muscle spasm for 20-30 minutes. The programme was supervised by a physiotherapist every other day and the parents were encouraged to continue exercises and ice massage at home. Treatment was carried out for 14 weeks, gradually reducing the number of visits to the physiotherapist.

Post treatment clinical assessment by inspection in the erect position showed no scoliosis, while the forward bending test showed only minimal scoliosis. Post treatment radiographs showed complete disappearance of the dorsal curve and a significant reduction of the lumber curve, the scoliosis angle being reduced to 12 degrees. (Figure 2) 


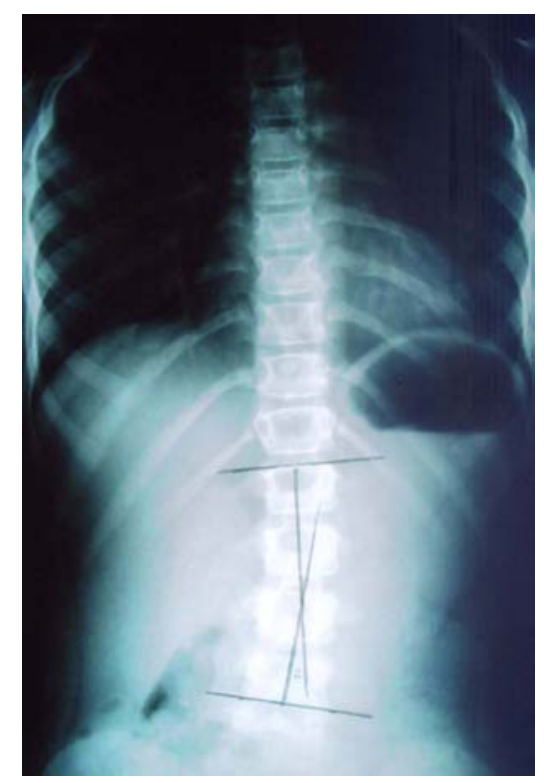

Figure 2

\section{Discussion}

Scoliosis of more than 10 degrees is considered abnormal $^{1}$. Scoliosis is described based on the direction of the convexity of the curve as a right or left curve. The most common pattern in idiopathic scoliosis is a right thoracic and left lumbar scoliosis ${ }^{1}$. Idiopathic scoliosis has been categorized, based on age of presentation as infantile (0-3 years), juvenile (3-10 years) and adolescent ( $>10$ years or from the onset of puberty to skeletal maturity $)^{1}$. This child had juvenile idiopathic scoliosis with a scoliosis angle of 20 degrees which reduced to 12 degrees after 14 weeks of physical therapy.

\section{Conclusion}

Early detection of scoliosis and proper conservative treatment with physical therapy can prevent the inevitable deformity and disability that occurs in later life.

\section{Acknowledgements}

I thank Dr. Sanjeewa Munasinha, Consultant Radiologist of Sri Lanka Army Medical Corps and Ms Rasika Abeyratne, Senior Physiotherapist of Sahana Rehabilitation Centre.

\section{References}

1. Boachie-Adjei O, Lonner B. Spinal deformity. Paediatric Clinics of North America 1996; 43: 883-96.

2. Weinstein SL. Idiopathic scoliosis: Natural history. Spine 1986; 11:780.

3. Nachemson AL, Peterson LE. Effectiveness of treatment with a brace in girls who have idiopathic scoliosis. J Bone Joint Surg Am 1995; 77: 823-7. 